\title{
MÁS ALLÁ DEL FEMINICIDIO \\ DE PAREJA: VICTIMIZACIÓN DE MUJERES POR HOMICIDIO Y CRIMINALIDAD ORGANIZADA TRANSNACIONAL EN EL PERÚ
}

Resumen: La victimización de mujeres ha sido pocas veces objeto de investigación criminológica en el Perú, dado el escaso desarrollo de la criminología en el país. No obstante, la política criminal que aborda la problemática de la violencia contra la mujer, mediante la tipificación del delito de feminicidio, amerita fundamentarse en este tipo de investigaciones. Como una primera aproximación, este artículo utilizó las mejoras en las estadísticas oficiales de homicidios, a fin de identificar y comparar variaciones temporales y espaciales en la victimización de hombres y mujeres, ocurridas entre los años 2011

\footnotetext{
Abogada de la Pontificia Universidad Católica del Perú, Máster en Criminología por la Universidad Católica de Lovaina y Máster Avanzado en Salud Pública y Desarrollo por la Universidad Libre de Bruselas y la Universidad Católica de Lovaina. Comisionada de investigación del Centro Nacional de Investigación Científica (CNRS) de Francia; Asesora de alta dirección del Ministerio de Justicia del Perú en materia de Política Criminal y Penitenciaria; Asesora de alta dirección del Ministerio del Interior del Perú en materia de Políticas de Seguridad y Orden Público. Lima, Perú. Correo-e: lucia.nunovero@pucp.edu.pe Fecha de recepción: 29 de diciembre de 2016. Fecha de modificación: 26 de mayo de 2017. Fecha de aceptación: 31 de agosto de 2017. Para citar el artículo: Lucía NuÑOVERo Cisneros. "Más allá del feminicidio de pareja: Victimización de mujeres por homicidio y criminalidad organizada transnacional en el Perú", en Revista Derecho Penal y Criminología, Vol. 38, n. ${ }^{\circ}$ 104, enero-junio de 2017, Bogotá, Universidad Externado de Colombia, pp. 243-265. DOI: https://doi.org/10.18601/ 01210483.v38n104.08
} 
y 2015. Mediante la aplicación de una escala, se encontraron los niveles más altos de victimización de mujeres por homicidio (de 5 a 15 muertes por 100 mil habitantes), en regiones con niveles altos o muy altos de victimización de hombres, tratándose además de regiones específicas de frontera o de desarrollo de criminalidad organizada transnacional, como minería ilegal, tráfico de drogas y trata de personas.

Palabras clave: Victimización, Mujeres, Criminalidad Organizada Transnacional, Perú, Homicidio.

\title{
BEYOND INTIMATE FEMICIDE: FEMALE HOMICIDE VICTIMIZATION AND TRANSNATIONAL ORGANIZED CRIME IN PERU
}

\begin{abstract}
The renovated academic interest on Criminology in Peru has offered little contribution to understand female victimization from a multidisciplinary perspective. Research in this field is required in order to discuss Criminal Policy initiatives to reduce violence against women in the country. This study approaches female victimization in Peru through official records of homicides from recent years. Regional and year variations in female and male homicide rates were identify and compared using an ad hoc scale. Higher levels of female homicide rates (5 to 15 homicides per 100,000 inhabitants) were found in regions with higher male homicide rates. Regions concerned were international border territories or regions characterized by widespread organized crime activities (illegal mining, drug trafficking, human trafficking, etc.).
\end{abstract}

Keywords: Victimization, Women, Organized Crime, Peru, Homicides, Official crime records.

\section{INTRODUCCIÓN}

En el año 2005, la Organización de las Naciones Unidas publicó un estudio epidemiológico que mostraba que el Perú mantenía altas tasas de violencia contra la mujer, con respecto a otros países del mundo ${ }^{1}$. Sobre la misma problemática, el Ministerio Público peruano emprendió, desde el año 2009, mejoras en el registro de denuncias de homicidios de mujeres y en el 2011, la modalidad de feminicidio fue tipificada

1 Organización Mundial de la Salud (OMS). Estudio multipaís de la oms sobre salud de la mujer y violencia doméstica contra la mujer, primeros resultados sobre prevalencia, eventos relativos a la salud y respuestas de las mujeres a dicha violencia: resumen del informe, 2005. 
como delito mediante la Ley n. ${ }^{\circ}$ 29819. Los últimos años, se ha llegado a afirmar que el Perú es el segundo país en acumular feminicidios en América Latina²

Sin embargo, queda un amplio campo para la investigación criminológica en lo referente a mujeres víctimas de delitos, así como de otros aspectos de la política criminal peruana relacionados con la violencia contra la mujer. Los estudios de victimización de mujeres son parte del necesario desarrollo de la criminología y la victimología en el Perú, así como de otras disciplinas que, aplicadas al fenómeno criminal, permiten sustentar de manera empírica la actualización del sistema jurídico penal peruano ${ }^{3}$.

Dentro del campo de la criminología, cabe resaltar la importancia de los estudios de tipo ecológico, cuyo objeto son los niveles de criminalidad que presenta una determinada población ${ }^{4}$. Dichos estudios permiten el diseño de políticas basadas en indicadores, como la tasa de homicidios, según variables de tiempo y espacio ${ }^{5}$. Sobre dicho indicador, tras la creación del Consejo Nacional de Política Criminal, el Ministerio de Justicia peruano constituyó un Comité Técnico que estandarizó la definición de muertes violentas asociadas a hechos dolosos, y completó y corrigió varios problemas de los registros policiales a través de encuestas y de comparaciones con los registros del Ministerio Público. Una tasa nacional de 6,5 homicidios por 100 mil habitantes fue inferida para el año 2012 y, mediante similares estimaciones, se infirieron tasas de homicidios y características del delito a nivel regional y provincial para los años 2011 a $2015^{[6]}$.

Ahora bien, la utilización de información oficial de la Policía y la Fiscalía para estimaciones de tasas delictivas no es novedosa, y cuenta con numerosos antecedentes en

2 Rocío Villanueva y Ministerio Público. Homicidio y feminicidio en el Perú. Lima, Observatorio de Criminalidad del Ministerio Público, 2009. Observatorio de Igualdad de Género de América Latina y el Caribe - CEPAL. Informe anual 2013-2014. El enfrentamiento de la violencia contra las mujeres en América Latina y el Caribe, Santiago de Chile, Naciones Unidas, 2015.

3 Klaus Roxin. Dogmática Penal y Política Criminal. Manuel Abanto Vásquez (trad.), Lima, IDEMSA, 1998, p. 25.

4 Elisa García EsPaÑa. Inmigración y delincuencia en España: análisis criminológico. Valencia, Tirant lo Blanch, Instituto Andaluz de Investigación Criminológica, 2001. Elías CARRANZA. "Situación del delito y de la Seguridad de los habitantes en los países de América Latina”, en Elías CARRANZA (coord.) Delito y seguridad de los habitantes. Costa Rica, ILANUd, Siglo XXI, 1997, pp. 23-49.

5 Oficina de las Naciones Unidas contra la Droga y el Delito (UNODC). Estudio Mundial sobre el homicidio 2013: Tendencias, contexto, datos.

6 Ministerio de Justicia y Derechos Humanos del Perú. Homicidios en el Perú: contándolos uno a uno 2012. Lima: Ministerio de Justicia y Derechos Humanos, 2014. Instituto Nacional de Estadística e Informática - Comité Estadístico Interinstitucional de la Criminalidad, Homicidios en el Perú: contándolos uno a uno 2011-2015. Lima, Instituto Nacional de Estadística e Informática, 2015. 
la criminología norteamericana ${ }^{7}$. Sin embargo, la reciente mejora de registros oficiales de delitos en el Perú permite, por primera vez, la discusión de teorías ecológicas del crimen, así como un mejor diseño de políticas de intervención en poblaciones específicas de mujeres ${ }^{8}$. Para ello, es necesario identificar las principales variaciones de la victimización de mujeres en el tiempo y según su distribución geográfica. Ello diferencia este estudio de aquellos que estiman la prevalencia o los factores de riesgo de violencia o victimización en poblaciones más reducidas como pacientes o comunidades nativas ${ }^{9}$.

En consecuencia, el presente estudio exploratorio pretende contribuir a la comprensión de la problemática de la victimización de mujeres en el Perú, especialmente por delitos violentos como homicidios. Además, busca aportar al debate suscitado por la reciente tipificación de la figura del feminicidio, discusión que en el Perú, como en otros países, requiere un enfoque criminológico interdisciplinario ${ }^{10}$.

\section{OBJETIVO}

El objetivo de este artículo es identificar las variaciones espacio-temporales de la victimización por homicidios de mujeres y hombres en el Perú. Ello nos permitirá, en primer lugar, caracterizar la victimización de mujeres en relación con el fenómeno

7 Robert O'Brien. "Police productivity and crime rates: 1973-1992", en Criminology, n. ${ }^{\text {3 } 34, ~ 1996,}$ p. 183. Colin Loftin y David McDowall, "The Use of Official Records to Measure Crime and Delinquency, Journal of Quantitative Criminology, 2010, vol. 26, no 4, pp. 527-532.

8 Felipe Hernando Sanz. "Eclecticismo y Diversidad en la Geografía del Crimen y la Delincuencia en el Cambio de Siglo/Eclecticism and diversity in the geography of crime and delinquency in the turn of the century/Éclectisme et diversité dans la géographie du crime et la délinquance dans le changement de siècle", en Anales de geografía de la Universidad Complutense, Vol. 26, enero 2006, Universidad Complutense de Madrid, 2006, pp. 9-30. CARlos VÁsquez GonzÁlez y Carles Soto URPINA. "El análisis geográfico del delito y los mapas de la delincuencia", en Revista de derecho penal y criminología, 2013, n. ${ }^{\circ}$ 9, pp. 419-448

9 DALlan F. FlaKe. "Individual, family, and community risk markers for domestic violence in Peru", en Violence against women, vol. 11, n. ${ }^{\circ}$ 3, 2005, pp. 353-373. SwEE MAY CRIPE et al. "Association of intimate partner physical and sexual violence with unintended pregnancy among pregnant women in Peru", en International Journal of Gynecology \& Obstetrics, vol. 100, n. . 2, 2008, pp. 104-108. HANS CONTRERAS-Pulache et al. "Características de la violencia durante el embarazo en adolescentes de Lima, Perú”, en Revista Peruana de Medicina Experimental y Salud Pública, vol. 30, n. . 3, 2013, pp. 379-385.

10 BeAtriz RamíRez. "Cuando la muerte se explica por el género: problematizando la tipificación del feminicidio/femicidio”, en Gaceta Constitucional, vol. 45, Lima, Gaceta Jurídica, 2011, pp. 353 360. Rocío GonzÁlez VelázQuEz. "Cuando el derecho penal no basta. Reflexiones en torno a la tipificación del feminicidio en México”, en Alegatos-Revista Jurídica de la Universidad Autónoma Metropolitana, May-Ago 2014, n. ${ }^{\circ}$ 87,pp. 271-308. Paola De la Rosa y Víctor Sandoval NavarRo. "Los Sesgos Cognitivos y su Influjo en la decisión judicial. Aportes de la psicología jurídica a los procesos penales de corte acusatorio", en Revista Derecho Penal y Criminología, Volumen XXXVII, n. ${ }^{\circ}$ 102, pp. 141-164, Universidad Externado de Colombia, junio 2016. 
de la violencia y la criminalidad del país. En segundo lugar, con base en nuestros resultados, discutiremos los alcances de la tipificación del feminicidio, como respuesta político-criminal al problema de la violencia contra la mujer en el país.

\section{METODOLOGÍA}

Se recolectó del Instituto Nacional de Estadística e Informática y del Comité Estadístico Interinstitucional de la Criminalidad, información sobre el número de hombres y mujeres víctimas de muertes violentas asociadas a hechos dolosos, ocurridas durante los años 2011-2015, en las 25 regiones del Perú. A partir de dichas cifras, hemos estimado las tasas de victimización por homicidio por cada cien mil habitantes, en las poblaciones de mujeres y hombres, proyectadas por el Instituto Nacional Estadística e Informática para dicho quinquenio ${ }^{11}$.

Mediante la construcción de series cortas de tiempo, identificamos las tendencias a nivel nacional de victimización por homicidios en las poblaciones estudiadas. Asimismo, a manera de un estudio ecológico, comparamos, mediante una escala de victimización (que distingue niveles de victimización bajo, intermedio, alto y muy alto), las variaciones de la victimización de mujeres y de hombres en las 25 regiones del Perú.

\section{RESULTADOS}

La tasa de muertes violentas asociadas a hechos dolosos por cien mil habitantes, llamada victimización por homicidios a efectos del presente estudio, es de 6 a 10 víctimas menos en la población femenina que en la masculina. Así también, a nivel nacional, para el año 2015, se estimó una ocurrencia de 2,6 muertes por cien mil habitantes en la población de mujeres y de 11,6 muertes por cien mil habitantes en la población de hombres. La variación (desviación estándar) es menor en la victimización de mujeres que en la de hombres, como se muestra en el Cuadro 1.

Por otro lado, para los años de estudio, se encontraron tasas de homicidios más elevadas tanto en población de mujeres como de hombres en las regiones de Madre de Dios y Tumbes. Entre las regiones de menor victimización de mujeres se encuentran las regiones Loreto y Apurímac, mientras que entre las de menor victimización de hombres, se encuentran las regiones de Huancavelica y Apurímac.

11 Instituto Nacional de Estadística e Informática- INEI, Perú. Estimaciones y proyecciones por sexo, según departamento, provincia y distrito 2000-2015, Lima, 2009. 


\section{Cuadro 1. Descriptivos de tasas regionales de homicidios en el Perú según sexo}

\begin{tabular}{cccll}
\hline \multicolumn{5}{c}{ Población mujeres } \\
\hline Año & Promedio (DS) & Mediana & \multicolumn{1}{c}{ Mínimo } & \multicolumn{1}{c}{ Máximo } \\
\hline 2011 & $2,3(1,5)$ & 1,9 & 0,6 (Loreto) & 7,6 (Madre de Dios) \\
2012 & $3,1(3,1)$ & 2,3 & 0 (Apurímac) & 9,6 (Túmbes) \\
2013 & $2,6(2,3)$ & 2,1 & 0,4 (Loreto) & 12,6 (Madre de Dios) \\
2014 & $2,9(2,7)$ & 2 & 0,3 (Ica) & 14,0 (Madre de Dios) \\
2015 & $3,1(1,3)$ & 3,1 & 0,8 (Loreto) & 6,8 (Madre de Dios) \\
\hline \multicolumn{5}{c}{ Población hombres } \\
2011 & $8,3(5,7)$ & 7,4 & 1,3 (Huancavelica) & 23,4 (La Libertad) \\
2012 & $10,7(7,6)$ & 9,3 & 0 (Apurímac) & 30,7 (Túmbes) \\
2013 & $11,7(9,6)$ & 8,3 & 2,1 (Moquegua) & 45,4 (Túmbes) \\
2015 & $12,4(8,9)$ & 9,6 & 2,4 (Huancavelica) & 40,9 (Túmbes) \\
\hline
\end{tabular}

Tasa por 100 mil habitantes hombres/mujeres.

A nivel de tendencia nacional, la victimización por homicidios se incrementó en 1,8 víctimas por cada cien mil habitantes, entre el 2011 y el 2015. De manera desagregada, este incremento fue de 0,7 en la población femenina, y de 2,7 en la población masculina. La tendencia creciente en la tasa de victimización por homicidios a nivel nacional se sitúa entre ambos indicadores como se muestra en el Gráfico 1.

Grafico 1. Tendencias de victimización por homicidio en Perú según sexo 2011-2015

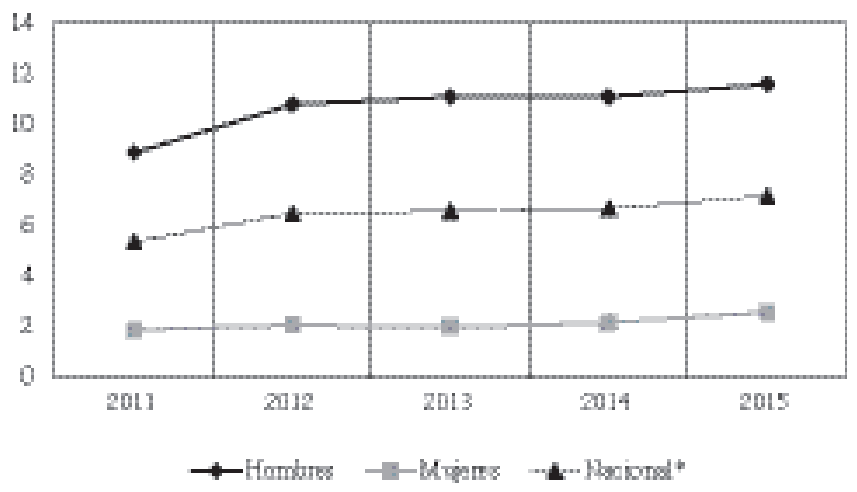

*La tasa a nivel nacional incluye víctimas no identificadas por sexo. 
No obstante la distribución de la población por sexo, mantiene proporciones equitativas a nivel nacional y regional (50,2 \% de la población del Perú estimada para el 2015 es de sexo masculino y 49,8\% de sexo femenino), la proporción de víctimas es diferente en cada región. El año 2015, se registraron mayores porcentajes de victimización de mujeres en la región de Lima y en las regiones de Puno, Arequipa y Cuzco. En conjunto, dichas muertes solo representaron el 8\% de los homicidios ocurridos ese año.

Finalmente, al aplicar una escala de cuatro niveles de victimización a las tasas estimadas para dicho período, se encontró que las variaciones regionales tienden a mantenerse a lo largo de los años, especialmente en regiones de alta y muy alta victimización, como Amazonas, Ancash, Cajamarca, Cuzco, Huánuco, Ica, Piura y San Martín, La Libertad, Madre de Dios y Tumbes.

\section{Cuadro 2. Tasas de victimización por homicidio en Perú según región y sexo}

\begin{tabular}{lcccccccccc}
\hline & \multicolumn{2}{c}{2011} & \multicolumn{2}{c}{2012} & \multicolumn{2}{c}{2013} & \multicolumn{2}{c}{2014} & \multicolumn{2}{c}{2015} \\
\hline & $\mathrm{H}$ & $\mathrm{M}$ & $\mathrm{H}$ & $\mathrm{M}$ & $\mathrm{H}$ & $\mathrm{M}$ & $\mathrm{H}$ & $\mathrm{M}$ & $\mathrm{H}$ & $\mathrm{M}$ \\
\hline Amazonas & 10,1 & 4,1 & 14,1 & 3,0 & 11,8 & 3,0 & 6,8 & 1,0 & 10,8 & 5,0 \\
Ancash & 9,0 & 2,3 & 16,8 & 2,3 & 17,7 & 2,1 & 18,3 & 1,8 & 11,5 & 3,5 \\
Apurímac & 4,8 & 1,8 & 0,0 & 0,0 & 6,9 & 3,1 & 5,2 & 0,9 & 4,7 & 3,1 \\
Arequipa & 8,3 & 3,2 & 9,1 & 3,4 & 9,1 & 2,1 & 7,3 & 3,0 & 6,6 & 3,4 \\
Ayacucho & 3,3 & 1,9 & 5,9 & 2,1 & 6,4 & 2,1 & 4,6 & 2,7 & 11,4 & 3,3 \\
Cajamarca & 9,4 & 0,9 & 11,8 & 2,8 & 10,3 & 2,0 & 11,5 & 1,6 & 10,9 & 2,6 \\
Callao & 18,9 & 1,3 & 22,1 & 2,4 & 25,1 & 1,6 & 28,0 & 1,4 & 26,2 & 3,5 \\
Cusco & 7,4 & 3,5 & 7,5 & 2,8 & 8,3 & 2,7 & 5,0 & 3,3 & 13,0 & 4,0 \\
Huancavelica & 1,3 & 1,7 & 2,9 & 1,7 & 3,3 & 0,8 & 2,4 & 2,0 & 6,8 & 2,8 \\
Huánuco & 9,7 & 2,2 & 10,1 & 1,9 & 7,2 & 1,2 & 11,1 & 4,0 & 14,9 & 4,7 \\
Ica & 6,9 & 1,9 & 9,7 & 1,3 & 11,1 & 1,3 & 13,0 & 0,3 & 12,9 & 3,1 \\
Junín & 5,1 & 2,2 & 5,7 & 1,8 & 5,2 & 1,4 & 5,6 & 2,3 & 5,9 & 2,8 \\
La Libertad & 23,4 & 2,1 & 26,7 & 3,7 & 27,3 & 3,8 & 25,1 & 2,9 & 21,1 & 1,9 \\
Lambayeque & 7,3 & 1,1 & 10,5 & 1,4 & 8,1 & 1,1 & 8,9 & 1,7 & 7,2 & 1,5 \\
Lima & 8,4 & 1,6 & 9,3 & 1,4 & 9,5 & 1,8 & 9,6 & 1,8 & 9,6 & 1,9 \\
Loreto & 2,7 & 0,6 & 3,4 & 0,4 & 3,8 & 0,4 & 4,3 & 1,6 & 6,3 & 0,8 \\
Madre de Dios & 18,2 & 7,6 & 17,7 & 14,7 & 21,3 & 12,6 & 24,7 & 14,0 & 27,9 & 6,8 \\
Moquegua & 2,2 & 2,5 & 3,2 & 1,2 & 2,1 & 2,4 & 8,4 & 1,2 & 5,2 & 3,6 \\
Pasco & 5,8 & 0,7 & 5,1 & 2,8 & 6,3 & 3,5 & 13,8 & 7,0 & 18,0 & 5,6 \\
Piura & 8,1 & 1,4 & 11,6 & 1,5 & 12,5 & 1,4 & 9,8 & 1,1 & 10,6 & 1,6 \\
Puno & 3,2 & 0,9 & 5,5 & 1,5 & 5,6 & 2,5 & 8,4 & 4,9 & 10,4 & 3,1 \\
San Martín & 18,3 & 4,1 & 18,2 & 3,0 & 16,4 & 2,9 & 13,5 & 3,4 & 19,5 & 3,1 \\
Tacna & 7,7 & 3,8 & 7,0 & 6,3 & 6,4 & 5,6 & 8,6 & 3,1 & 9,0 & 1,8 \\
Tumbes & 5,7 & 3,9 & 30,7 & 9,6 & 45,4 & 2,8 & 40,9 & 1,9 & 34,9 & 1,8 \\
Ucayali & 4,4 & 1,8 & 7,1 & 3,6 & 6,6 & 1,8 & 10,8 & 2,6 & 13,7 & 4,7 \\
\hline
\end{tabular}

Nivel de victimización: Bajo (de 0 a 5), Intermedio (de 5 a 10), Alto (de 10 a 20) y Muy alto (más de 20 homicidios por cien mil habitantes). 
Dichas variaciones regionales, según la escala elaborada, muestran también diferencias en la brecha de victimización de mujeres y de hombres por homicidio. No se encontraron niveles muy altos de victimización de mujeres en ninguna de las 25 regiones. Ahora bien, los niveles más altos de victimización en población femenina se observan en la región de Madre de Dios y Tumbes, dos de las cuatro que presentan niveles muy altos de victimización en hombres. Lo opuesto no ocurre y existen regiones como La Libertad y Callao, en las que se dan altos niveles de victimización de hombres, acompañados de bajos niveles de victimización de mujeres. También, se encontró que los niveles intermedios de victimización de mujeres, hallados en las regiones de Amazonas, Pasco y Tacna, se acompañaron también de niveles intermedios y altos de victimización de hombres por homicidio.

\section{DISCUSIÓN}

\section{La importancia de los estudios de sesgos en criminología y los riesgos de la política criminal peruana}

Como hemos dicho, en el Perú abundan reportes institucionales y literatura gris que recoge mediciones de delitos y hechos de violencia contra la mujer. Sin embargo, se requieren investigaciones criminológicas aplicadas al desarrollo del sistema jurídico penal peruano en lo concerniente a la mujer como víctima de delitos o violencia.

Antes de discutir los resultados del presente estudio, consideramos necesario precisar el camino seguido por la criminología contemporánea en el Perú, y recalcar la importancia de los estudios de sesgos para las futuras investigaciones en la materia. En general, como parte de una necesaria línea de investigación en criminología cuantitativa, y en particular, para la elucidación de sesgos en la construcción de figuras como el feminicidio, cuyo proceso involucra la participación de medios de comunicación, en el marco de lo que podría ser considerada una búsqueda de efectos simbólicos en la sociedad, mediante figuras del Derecho penal.

Para empezar, hemos de notar, que en el Perú, la pregunta sobre el crimen y el criminal ha sido aproximada en primer lugar desde la cárcel. Los más recientes y rigurosos estudios que contribuyen a la comprensión del fenómeno criminal no solamente del país, sino de América Latina, se han realizado a partir de las personas recluidas en establecimientos penitenciarios, desde perspectivas como la histórica ${ }^{12}$ y la etno-

12 Ricardo Salvatore, Carlos Aguirre y Gilbert Joseph (eds.). Crime and punishment in Latin America: law and society since late colonial times. Durham y Londres, Duke University Press, 2001. Carlos Aguirre. The Criminals of Lima and Their Worlds: The Prison Experience, 1850-1935. Durham y Londres, Duke University Press, 2005. 
gráfica, a través de la cual se han descrito los grupos, organizaciones y dinámicas de poder que conforman los espacios carcelarios ${ }^{13}$.

Ahora bien, durante los últimos años también han surgido no solo en Perú, sino en América Latina, estudios enfocados en mediciones de incidencia de delitos, que no necesariamente se dirigen a ahondar en teorías o análisis específicos al objeto criminal, sino al desarrollo de políticas públicas. Dichos estudios establecen, por ejemplo, relaciones entre la violencia o el crimen y los niveles de confianza institucional o interpersonal en países como el Perú, y se sirven de indicadores como las tasas de homicidios ${ }^{14}$. Se trata entonces de recientes investigaciones a vocación aplicada, que involucran mediciones de delitos y enfrentan por lo menos dos riesgos metodológicos:

En primera instancia, el riesgo de encontrar problemas de falseamiento o manipulación de registros de delitos. Este problema podría ser trazado en el Perú, desde la utilización de estadísticas policiales en materia de tráfico de drogas, en la década de los noventa. Durante dicha época, la expansión de los medios de comunicación coincidió con un fenómeno de populismo en el uso de los temas de seguridad en el Perú ${ }^{15}$.

Como señalan Díez Ripollés y otros autores a propósito de las nuevas tendencias político-criminales, el protagonismo de los medios de comunicación en la discusión pública de temas sociales, así como su conformación como agentes de control social, comporta una consolidación comunicativa y discursiva bastante positiva para la sociedad. Sin embargo, también conlleva el riesgo del falseamiento o empobrecimiento de la información por intereses mercadotécnicos, así como por desconocimiento u ocultamiento de datos relevantes ${ }^{16}$.

Además, existe un segundo riesgo metodológico específico, al cual se enfrentan estudios como el presente. Se trata del problema de abordar, desde una perspectiva marcadamente cuantitativa, fenómenos poco estudiados y que, por lo tanto, no cuentan con un adecuado marco teórico, que clarifique conceptos y variables, dado el escaso desarrollo de específicas parcelas de la criminología contemporánea en el Perú.

13 José Luis Pérez Guadalupe. La construcción social de la realidad carcelaria. Lima, Pontificia Universidad Católica del Perú, Fondo, 2000. José Luis Pérez Guadalupe. Faites y atorrantes. Una etnografía del penal de Lurigancho, Lima, Centro de Investigaciones Teológicas, 1994.

14 Patricia Zárate, Jorge Aragón y Jorge Morel. Inseguridad, estado y desigualdad en el Perú y América Latina: un estado de la cuestión, Lima, Instituto de Estudios peruanos, 2013.

15 Ignacio Berdugo Gómez de la Torre, CARmen Gómez Rivero y AdÁn Nieto Martín. “El sistema penal y penitenciario peruano. Reflexiones político-criminales", en América Latina hoy, Vol. 28, 2001, pp. 19-47. LuCía Nuñovero Cisneros. "Política criminal actuarial y tráfico de drogas en el Perú de los noventas”, en Revista Internacional Derecho Penal Contemporáneo, Vol. 31, abr-jun. 2010, pp. 43-80. Bogotá, Legis, 2010.

16 José Luis Díez RiPollés, Estudios Penales y de Política Criminal, Lima: IDEMSA, 2007, pp. 55, ANDRÉ LuIS CALlEGARI. "Crimen organizado: concepto y posibilidad de tipificación delante del contexto de la expansión del derecho penal”, en Derecho Penal y Criminología, 2010, vol. 31, p. 15. 
En este sentido, los estudios que identifican la existencia de sesgos han aportado mucho a la criminología, mostrando, por ejemplo, que el sesgo de reportabilidad es menor en el delito de homicidio con respecto a otros delitos, puesto que los homicidios por su gravedad tienden a ser casi siempre denunciados y registrados por la Policía y la Fiscalía. La naturaleza de este delito, además, le hace susceptible de ser registrado por los servicios de salud, acrecentando su fiabilidad. Como parte de una línea de investigación sobre mediciones de delitos en base, es necesaria una especial atención a este u otro tipo de sesgos o artefactos que invalidan los resultados de las mediciones de delitos efectuadas en el Perú ${ }^{17}$.

Así, el que algunos autores hayan planteado la utilidad de indicadores agregados de feminicidio y tentativa de feminicidio en el Perú ${ }^{18}$, ha ido en contra de un consenso científico de bastante data, que, como hemos dicho, privilegia al homicidio como indicador, sobre otros delitos, o grados de consumación delictiva, en mérito a su menor sesgo de reportabilidad.

Sin embargo, lo mencionado no agota la discusión acerca de la validez y utilidad de las mediciones de violencia contra la mujer, basadas en ocurrencias de delitos específicos, como pueden ser homicidios o feminicidios. Por nuestra parte, consideramos que dadas las múltiples dimensiones y formas que dicha violencia adopta, y que en particular algunas, como la violencia psicológica, pueden resultar más sutiles aunque no por ello menos dañosas, es necesario que el desarrollo de indicadores incorpore aproximaciones cualitativas, como es propio a un marco conceptual criminológico y a una respuesta estatal que excede lo penal ${ }^{19}$.

Así, desde un punto de vista metodológico, parece poco probable la reciente construcción de indicadores fiables de feminicidios en el Perú. La interferencia política y la distorsión de indicadores en delitos como el feminicidio, ya ha sido destacada por algunos autores en España y América Latina ${ }^{20}$. En nuestros términos, el problema

17 Ted R. GurR. "Historical trends in violent crime: A critical review of the evidence", en Crime and Justice, 1981, pp. 295-353. STEVEn LeVITT. “The Relationship between Crime Reporting and Police: Implications for the use of Uniform Crime Reports", en Journal of Quantitative Criminology, 1998, vol. 14,n. ${ }^{\circ}$ 1, pp. 61-81. Colin LofTin y David MCDOwall. "The Use of Official Records to Measure Crime and Delinquency”, en Journal of Quantitative Criminology, 2010, vol. 26, n. ${ }^{\circ}$ 4, pp. 527-532

18 Wilson HeRnández Breña. "Feminicidio (agregado) en el Perú y su relación con variables macrosociales", en URVIO-Revista Latinoamericana de Seguridad Ciudadana, n. ${ }^{\circ}$ 17, 2015, FLACSO - Ecuador (Facultad Latinoamericana de Ciencias Sociales), pp. 48-66.

19 Enrique Odriozola, Javier Fernández Montalvo y PaZ de Corral Gargallo. “¿Hay diferencias entre la violencia grave y la violencia menos grave contra la pareja?: un análisis comparativo", en International Journal of Clinical and Health Psychology, Vol. 8, n. ${ }^{\circ}$ 2, Asociación Española de Psicología Conductual, 2008, pp. 355-382.

20 Raquel Osborne. "De la 'violencia' (de género) a las 'cifras de la violencia': una cuestión política”, en EMPIRIA. Revista de Metodología de Ciencias Sociales, 2008, no 15, p. 99-124. JARIS MuJICA, J. y DiEgo TUESTA. "Problemas de construcción de indicadores criminológicos y situación comparada 
metodológico de las mediciones de feminicidio en el Perú radica en que dichos registros y mediciones surgen en un contexto de campaña que promueve mayor denuncia de un delito, y que por ende favorece la aparición de falsos positivos.

En efecto, los estudios de sesgos en criminología abordan el problema del tratamiento que dan los medios de comunicación a los delitos, teniendo en cuenta los fenómenos de pánico moral, miedo al crimen o populismo penal que, como hemos dicho, implican también el uso de estadísticas criminales y manipulación de información con fines publicitarios y políticos ${ }^{21}$. Así, por ejemplo, los estudios de la distorsión de resultados de encuestas de victimización por aparición de delitos en televisión presentan una interesante alternativa para persistir en la construcción de una política criminal basada en evidencia en países como el Perú ${ }^{22}$. Consideramos, por ello, que es importante desarrollar este tipo de estudios dentro de una línea de investigación criminológica cuantitativa, campo, por lo demás, bastante prometedor para el desarrollo de la disciplina en el Perú.

\section{Perspectivas de victimización y problemas de los estudios de victimización en el Perú}

En segundo lugar, cabe precisar las ventajas y límites de la perspectiva y corpus utilizados en el presente artículo. Nosotros desarrollamos nuestro cuestionamiento escogiendo, entre las diferentes perspectivas que ofrece la criminología contemporánea, una perspectiva positivista, que enfoca principalmente mediciones de víctimas, daños o características del delito, así como tipologías y explicaciones construidas en base a dichas mediciones ${ }^{23}$.

Existen otras perspectivas que abordan la victimización, como un proceso que comprende etapas de experiencia de victimización, percepción de injusticia, búsqueda $\mathrm{y}$, finalmente, consecución de una validación de la víctima en tanto ser humano ${ }^{24}$. Y de hecho, todavía existen otras posibilidades de abordar el fenómeno de la victimización de mujeres, que se han dejado de lado en este estudio.

del feminicidio en el Perú”, en Anthropologica, Vol. 30 n. ${ }^{\circ}$ 30, Pontifica Universidad Católica del Perú, 2012, pp. 169-194.

21 Kenneth Thompson. Moral panics. Nueva York, Routledge, 1998. Flemming Balvig et al. "The public sense of justice in Scandinavia: A study of attitudes towards punishments", en European Journal of Criminology, vol. 12, no 3, pp. 342-361.

22 Justin Baer y William J. Chambliss. "Generating fear: The politics of crime reporting”, en Crime, Law and Social Change, 1997, vol. 27, no 2, p. 87-107. Donald L. Diefenbach y Mark D. West. "Violent crime and Poisson regression: A measure and a method for cultivation analysis", en Journal of Broadcasting \& Electronic Media, 2001, vol. 45, n. ${ }^{\circ} 3$, pp. 432-445.

23 Mitchell J. Miller (ed.). The Encyclopedia of Theoretical Criminology, Vol. 1, Nueva Jersey, John Wiley \& Sons, 2014.

24 Hilda Marchiori. Criminología: La víctima del delito, México, Porrúa, 1998. 
Ahora bien, desde la perspectiva adoptada, en el Perú contamos con encuestas de victimización, metodología considerada como una de las áreas más prometedoras para "cuantificar" la delincuencia y su evolución a nivel internacional ${ }^{25}$. Sin embargo, la utilización de registros de homicidios para nuestra investigación se sustenta en las limitaciones de las cuales aún adolecen dichas encuestas de victimización en el Perú. No nos referimos solamente a problemas metodológicos relacionados con el tipo de encuesta auto-declarada, los errores de muestreo, de cuestionario o de registro poblacional, sino a la poca idoneidad de las encuestas para la medición de ciertos delitos específicos, como homicidios o violencia familiar. En efecto, la fiabilidad de las encuestas es menor en delitos contra bienes jurídicos colectivos, así como en delitos en los que puede existir un elemento de cercanía familiar. En el caso del delito de homicidio, la encuesta tendría que recoger información ya no de la víctima, sino de manera indirecta a través de un familiar de esta ${ }^{26}$. Todo ello sin contar que existen, como hemos dicho, efectos distorsionadores en dichas encuestas generados por los medios de comunicación, máxime en momentos en que la opinión pública se ha ocupado especialmente del problema de la violencia contra la mujer ${ }^{27}$.

En el Perú, dos instituciones públicas han elaborado encuestas de victimización: el Instituto Nacional de Estadística e Informática (INEI), mediante la Encuesta Nacional de Programas Estratégicos (ENAPRES), y El Ministerio Público, ambas bajo metodologías diferentes y sin alcanzar estimaciones a nivel de la población total nacional ${ }^{28}$. La sociedad civil ha contribuido con estudios escasamente replicables y con mayor énfasis en la victimización subjetiva o percepción de inseguridad ${ }^{29}$.

En consecuencia, caracterizar la victimización de mujeres mediante los hallazgos de muertes violentas asociadas a hechos dolosos, permite minimizar sesgos y aproxi-

25 Anna del Frate. "Crime and criminal justice statistics challenges", en Stefan HaRRendorf, MarkKu Heiskanen y Steven Malby (eds.). Internation Statistics of Justice and Crime. Helsinki, Heuni, 2010 p. 168.

26 Steven Lab et al. Criminal Justice: The Essentials, Oxford, Oxford University Press, 2013.

27 Justin Baer y William J. Chambliss. "Generating fear: The politics of crime reporting". Crime, Law and Social Change, 1997, vol. 27, n. ${ }^{\circ}$, p. 87-107. Donald L. Diefenbach y Mark D. West. "Violent crime and Poisson regression: A measure and a method for cultivation analysis", en Journal of Broadcasting \& Electronic Media, 2001, vol. 45, n. ${ }^{\circ}$ 3, pp. 432-445.

28 La ENAPRES de mayor alcance elabora mediciones con base en semestres móviles de victimización por una serie de delitos en población urbana de 15 a más años de edad. Instituto Nacional de Estadística e Informática. Encuesta Nacional de Programas Estratégicos, Lima, INEI, 2016.

29 A diferencia de una victimización objetiva de un hecho del cual el ciudadano ha sido víctima, la victimización subjetiva describe la percepción de que se es susceptible de ser víctima de un delito a futuro, preguntó "si pensando en la posibilidad de ser víctima de un delito, se siente seguro, algo seguro, algo inseguro o muy inseguro", mientras que la ONG Latinobarómetro se interesa en medir victimización familiar cuando pregunta “¿Ha sido usted o algún pariente asaltado, agredido, o víctima de un delito en los últimos doce meses?". Ciudad Nuestra, Segunda Encuesta Metropolitana de Victimización 2012 - Resultados en 35 distritos de Lima, Lima, Ciudad Nuestra, 2012. 
marnos directamente a las víctimas de este delito especialmente violento. En segundo lugar, el corpus elegido permite relacionar dicha victimización con un fenómeno más amplio, como es el de la violencia y el crimen en el Perú, mediante una metodología estandarizada a nivel provincial, regional y nacional.

\section{Homicidios de mujeres y criminalidad organizada transnacional en el Perú}

Nuestro análisis exploratorio muestra que, durante el período estudiado, la victimización en mujeres fue menor a la de hombres, en lo que respecta a homicidios, delito caracterizado por la violencia de los medios empleados y la irreversibilidad de sus efectos en la víctima y su entorno. Estudios similares realizados en México y Colombia también resaltan la brecha de victimización por homicidios entre hombres y mujeres, y el mayor riesgo que enfrenta la población masculina de ser víctima de este delito. Recientes estudios han intentado relacionar dichas diferencias con los roles de género ${ }^{30}$, en el marco de estudios más generales que explican la tendencia masculina al comportamiento violento ${ }^{31}$.

Sin embargo, más allá de dicha diferencia, la mayoría de estos estudios concuerdan en la importancia de la variable geográfica del crimen, aspecto relacionado con los postulados de la Escuela Criminológica de Chicago, conocidos como las teorías ecológicas del crimen ${ }^{32}$. De la misma manera, nuestros hallazgos exploratorios muestran la necesidad de identificar factores criminógenos o protectores en determinadas regiones y localidades en las que se encuentran altos niveles de victimización de mujeres por homicidio. En el Perú, nuestros hallazgos sugieren que se requieren

30 MARÍA López et al. "Muertes por homicidio, consecuencia fatal de la violencia: El caso de México, 1979-1992”. Revista de Saúde Pública, 1996, vol. 30, n. ํ 1, p. 46-52. RicArdo PedraZA, PAOla NeIRA y YAHIRA G. SABOGAL, "Muertes violentas intencionalmente producidas en Bogotá, 1997-2005: diferencias según el sexo”, en Revista Colombiana de Psiquiatría, 2008, vol. 37, n. 3, p. 316-329. Rosemary Gartner, Kathryn BaKer y Fred PAMPEl. "Gender stratification and the gender gap in homicide”, en Social Problems, 1990, vol. 37, n. 4, pp. 593-612. JEAn Dreze y ReETIKa KHERA. "Crime, gender, and society in India: Insights from homicide data", en Population and development review, 2000, vol. 26, n. ${ }^{\circ}$ 2, pp. 335-352. JANET P. STAMATEL. "Explaining variations in female homicide victimization rates across Europe", en European Journal of Criminology, 2014, vol. 11, n. ${ }^{\circ}$ 5, pp. 578-600.

31 AnNe CAmpbell. "Sex differences in direct aggression: What are the psychological mediators?", en Aggression and Violent behavior, 2006, vol. 11, n. ${ }^{\circ} 3$, pp. 237-264. RYAN SCHACHT, R., KRISTIN Rauch y MoniQue Mulder. "Too many men: the violence problem?”, en Trends in Ecology \& Evolution, vol. 29, n. ${ }^{\circ} 4,2014$, pp. 214-222.

32 Clifford Robe Shaw, Henry Donald McKay y Norman S. HAYNER. Juvenile delinquency and urban areas: A study of rates of delinquents in relation to differential characteristics of local communities in American cities. Chicago, University of Chicago Press, 1942. NicHOLAR EMERICK et al. "Homicide and social disorganization on the border: Implications for Latino and immigrant populations", Social Science Quarterly, Vol. 95, .n 2, Southwest Social Science Association, 2014, pp. 360-379. 
estudios en zonas que presentan dinámicas de criminalidad organizada transnacional, como tráfico de drogas, minería ilegal, trata de personas y contrabando.

En efecto, en el Perú, la presencia de niveles altos de homicidios en población femenina se ve acompañada de los niveles máximos de victimización por homicidios en población masculina, en las regiones específicas de Madre de Dios y Tumbes. Estudios enfocados a estas regiones de frontera permitirían elucidar si se trata de una problemática particular de criminalidad organizada de nivel transnacional o de desorganización social relacionado con dinámicas fronterizas.

En efecto, por ejemplo Madre de Dios se ha caracterizado los últimos años por un creciente problema de minería ilegal y trata de personas ${ }^{33}$, mientras que Tumbes se encuentra como paso de frontera a Ecuador, en una de las rutas de cocaína más importantes del país. Así también, la victimización por homicidio de mujeres, acompañada por niveles intermedios y altos de victimización de hombres, se encontró en regiones como Pasco y Tacna, que muestran una participación en la economía de la cocaína a través de cultivos o de rutas de droga y contrabando ${ }^{34}$.

Dichos resultados sugieren la necesidad estudios de caso, y comparados con regiones, cuyos niveles altos de victimización de hombres y criminalidad organizada no ha significado una mayor victimización de mujeres. Es el caso de las regiones de Callao y La Libertad, en donde se concentra el negocio de la cocaína, de exportación y producción respectivamente, así como de criminalidad organizada, relacionada con el sector construcción que ha alcanzado especialmente durante los últimos años, un nivel de interconexión macro-regional.

Así, siguiendo la literatura actual, la problemática a analizar en las cinco únicas regiones que presentan intermedios o altos niveles de victimización de mujeres implica factores socioeconómicos, de composición poblacional u otros que puedan influir en la elevada victimización de mujeres en ciertas regiones ${ }^{35}$. Por otro lado,

33 Pablo de la Cuba, Fabián Novak y SAndRa Namihas. La trata de personas con fines de explotación laboral en el Perú. El caso de la minería aurífera y la tala ilegal de madera en Madre de Dios. Lima, Instituto de Estudios Internacionales (IDEI) de la Pontificia Universidad Católica del Perú y la Organización Internacional para las Migraciones (OIM), 2009. ANTONIo BRACK et al. Minería Aurífera en Madre de Dios y Contaminación con Mercurio - Una Bomba de Tiempo, Lima, Ministerio del Ambiente, 2011.

34 Naciones Unidas Oficina contra la Droga y el Delito (UNODC). Drogas y Delitos en el Perú: Situación actual y Evolución, Informe 2007. Lima: Naciones Unidas contra la Droga y el Delito, 2007. Comisión Nacional para el Desarrollo y Vida Sin Drogas (DEVIDA). Estrategia Nacional de Lucha contra las Drogas 2012-2016. Lima: Comisión Nacional para el Desarrollo y Vida Sin Drogas, 2016.

35 Kenneth C. Land, Patricia L. McCall y Lawrence E. Cohen. "Structural covariates of homicide rates: Are there any invariances across time and social space? ”, en American journal of sociology, Vol. 95, n. ${ }^{\circ} 4$, Enero 1990, The University of Chicago Press, 1990, pp. 922-963. PATrick W. O'CARroll y JAMES A. MERCY. "Regional variation in homicide rates: why is the West so violent?", en Violence 
nuestros hallazgos muestran la necesidad de sobrepasar dicho marco de análisis hacia el estudio de dinámicas, espacios e instituciones vinculadas a la criminalidad organizada, así como de rutas y mercados ilegales transnacionales relacionados con dicha victimización ${ }^{36}$.

\section{Feminicidio: emprendimiento moral y formas de violencia contra la mujer}

Finalmente, a propósito de la reciente incorporación del delito de feminicidio al Código Penal peruano, al cual le corresponden penas de más de 15 y 25 años de prisión, es importante partir de la premisa de que los homicidios de mujeres constituyen expresiones especialmente graves de la problemática de la violencia, y en particular de la violencia contra la mujer, existente en la sociedad peruana.

En efecto, consideramos que el aporte fundamental de la criminología a esta problemática es el contribuir a una mejor comprensión de las diferentes formas que toma la violencia contra la mujer y de los delitos en los cuales estas se manifiestan. En consecuencia, los futuros estudios acerca del feminicidio en el Perú se han de dirigir a desarrollar tipologías y adoptar una mayor precisión terminológica acerca de las específicas formas de homicidio que implica víctimas mujeres, sobre las cuales se desarrollan aproximaciones científicas, a finalidad político criminal.

En particular, resulta de mucha utilidad denominar feminicidio íntimo o de pareja ${ }^{37}$, a aquellos supuestos relacionados con la violencia íntima o de pareja, distinguiéndolos así de los homicidios de mujeres de tipo comunitario o ecológico, que podrían estar relacionados con delitos como la trata de personas, las violaciones sexuales o a la criminalidad organizada, como sugiere la presente investigación. En la actualidad, la doctrina peruana aún no desarrolla esta importante distinción y ambas problemáticas se encuentran recogidas en el art. 108-B del Código Penal peruano, que tipifica el delito de feminicidio.

and victims, vol. 4, n. ${ }^{\circ}$ 1, 1989, pp. 17-25. PATRICIA L. MCCALL et al. "Explaining variation in homicide rates across Eastern and Western European cities: the effects of social, political, and economic forces", en Handbook of European Homicide Research, Nueva York, Springer New York, 2012, pp. 137-154.

36 DANiel SAnsó-Rubert. "Nuevas tendencias de organización criminal y movilidad geográfica. Aproximación geopolítica en clave de inteligencia criminal"/Tìtulo en Inglés: "New Tendencies in Criminal Organization and Geographical Mobility. Geopolitical Approach and Criminal Intelligence", en UNISCI Discussion Papers, 2016, no 41, p. 181. LiosdAY LANDABURo SÁnCHEZ. Crimen organizado y economía ilegal. URVIO-Revista Latinoamericana de Estudios de Seguridad, 2016, n. ${ }^{\circ}$ 18, p. 125-136.

37 Yuliana Vélez Guzmán. "Femicides in Medellin, 2010-2011: Conceptualization, characterization, and analysis", en Revista Criminalidad, 2012, vol. 54, n. ${ }^{\circ}$, pp. 13-26. 
Además, abordar la problemática del feminicidio íntimo o de pareja en su especificidad frente a otro tipo de fenómenos ecológicos permite una mejor reflexión criminológica acerca de la efectividad de la creación de nuevos tipos penales, que podrían ser fruto de lo que Díez Ripollés denomina un Derecho penal simbólico o efectista.

En efecto, la tipificación del feminicidio, en sociedades como las peruanas, en las que la violencia de pareja o entre familiares ha tendido a ser vista como normal, adquiere relevancia político criminal, pese a que no haya podido fundamentarse en términos cuantitativos, de manera rigurosa. Así, desde un punto de vista criminológico, las leyes n. ${ }^{\circ} 29819$ del 2011 y n. ${ }^{\circ} 30068$ del 2013, que establecen el feminicidio como tipo penal específico, podrían caracterizarse como un emprendimiento moral, similar al que describe Becker para el caso de la prohibición de la marihuana en Estados Unidos ${ }^{38}$. Ello explica el importante rol que cumplen dentro de dicho emprendimiento moral, las campañas mediáticas que buscan sensibilizar a la opinión pública sobre el problema de la violencia contra la mujer y el rechazo que ha generado la impunidad de casos en los que aparecen figuras mediáticas, como víctimas de violencia de pareja ${ }^{39}$.

Ahora bien, proseguir la investigación a este respecto requiere discutir como hipótesis que la homogeneización de la opinión pública en torno a un consenso normativo es lo que ha permitido la creación e implementación de la ley del feminicidio en el Perú. A su vez, implica la necesidad de estudios socioantropológicos y culturales que se interesen, entre otros, en las actitudes paternalistas de los actores del sistema penal, la utilización de recursos para implementar agendas morales o en la creación de pánicos sociales en la sociedad peruana.

De esta manera, los futuros estudios acerca del feminicidio en el Perú podrían también enfocarse en la construcción de etiquetas sociales, tratándose este de uno de los mayores aportes de Becker a la criminología contemporánea. En efecto, los estudios acerca de las identidades y figuras de víctimas que utilizan los medios de comunicación y los actores políticos, han encontrado una proclividad a construir “inocentes" o responsables de su propia victimización ${ }^{40}$. Esta importante discusión permite considerar la dimensión terapéutica de la incorporación de las víctimas a la sociedad. Finalmente, caracterizar la tipificación del feminicidio como un emprendimiento moral, en el cual participan diferentes actores de la política criminal peruana,

38 HowARD BeCKer. Outsiders. Hacia una sociología de la desviación, Siglo XXI, Buenos Aires, 2009.

39 FAnNi MuÑoz CABREJo. "Discursos sobre el feminicidio en la prensa escrita: El Comercio (2012) y Trome (2013): entre la visibilización/invisibilización del fenómeno”, en Intersticios de la política y la cultura. Intervenciones latinoamericanas, 2016, vol. 5, n. ${ }^{\circ}$ 9, pp. 97-125. VíctOR MONTERO. "Análisis psicosocial del discurso de la prensa sensacionalista peruana y las actitudes de sus lectores". Revista de investigación en psicología, 2008, vol. 11, n. ${ }^{\circ}$ 2, pp. 153-181.

40 Michelle Meloy y Susan Miller. The victimization of women: Law, policies, and politics. Nueva York, Oxford University Press, 2010. 
permite una reflexión acerca de los instrumentos a través de los cuales la psicología criminológica busca que los individuos accedan a un estatus de no-víctima, y que sobrepasando el hecho criminal, se desarrollen plenamente en la sociedad.

\section{CONCLUSIONES}

La victimización de mujeres por homicidios en el Perú, a pesar de presentar niveles, tendencias de aumento y variaciones menores a la de hombres en las 25 regiones del país, podría estar relacionada con los altos niveles de violencia y criminalidad que caracterizan ciertas regiones de frontera, o en las que se reportan dinámicas de criminalidad organizada transnacional. Es necesario, por ello, emprender estudios de caso en dichas regiones, dentro de una línea de estudios ecológicos, que rinda cuenta de las variaciones espacio-temporales, así como de los diferentes factores y dinámicas que explican los altos niveles de victimización encontrados.

Los alcances de la reciente criminalización de la figura del feminicidio, como respuesta político-criminal frente al problema de la violencia contra la mujer en el Perú, son aún limitados. El principal problema que reviste la figura es el no distinguir suficientemente supuestos de violencia de pareja o íntima, de supuestos relacionados con violencia comunitaria o ecológica como la presente, en zonas o regiones de alta criminalidad.

En consecuencia, en cuanto al futuro de la investigación criminológica aplicada al desarrollo de políticas y legislación penal relacionada con la violencia contra la mujer, consideramos de primer orden que se desarrollen dos líneas de investigación que la sustenten: en primer lugar, una línea de estudios de sesgos en las mediciones de delitos, que sistematice e incorpore el manejo de sesgos en las diferentes mediciones elaboradas por instituciones penales y de la sociedad civil, en cuya agenda puedan primar la mercadotecnia o el sesgo institucional al rigor científico; en segundo lugar, se requieren investigaciones antropológicas y culturales en torno a la construcción de víctimas o agresores en medios de comunicación, incluyendo víctimas de violencia contra la mujer.

Por último, consideramos necesario continuar la presente exploración y análisis de las variaciones espacio-temporales en la ocurrencia de delitos en el Perú, así como incorporar a la reflexión criminológica y político criminal peruana, instrumentos cuantitativos mucho más potentes que los aquí perfilados. Dichos desarrollos académicos, así como los estudios de caso de zonas de alta criminalidad, como Tumbes, Madre de Dios, Tacna, Callao y La Libertad, permitirán mejorar las políticas y legislaciones relacionadas con el problema de la violencia y la delincuencia en el Perú. 


\section{BIBLIOGRAFÍA}

Aguirre, CArlos. The Criminals of Lima and Their Worlds: The Prison Experience, 1850-1935. Durham y Londres, Duke University Press, 2005.

Baer, Justin y Chambliss, William J. "Generating fear: The politics of crime reporting”, en Crime, Law and Social Change, 1997, vol. 27, n. ${ }^{\circ}$ 2, p. 87-107.

BAer, Justin y Chambliss, William J. "Generating fear: The politics of crime reporting”. Crime, Law and Social Change, 1997, vol. 27, n. ${ }^{\circ}$ 2, p. 87-107.

BeCKer, HowARD. Outsiders. Hacia una sociología de la desviación, Siglo XXI, Buenos Aires, 2009.

Berdugo Gómez de la Torre, Ignacio; Gómez Rivero, CARMEn y Nieto Martín, ADÁn. "El sistema penal y penitenciario peruano. Reflexiones político-criminales", en América Latina hoy, Vol. 28, 2001, pp. 19-47.

Brack, Antonio et al. Minería Aurífera en Madre de Dios y Contaminación con Mercurio - Una Bomba de Tiempo, Lima, Ministerio del Ambiente, 2011.

CAllegari, André Luis. "Crimen organizado: concepto y posibilidad de tipificación delante del contexto de la expansión del derecho penal", en Derecho Penal y Criminología, 2010, vol. 31, p. 15.

CAMPBEll, AnNE. "Sex differences in direct aggression: What are the psychological mediators?", en Aggression and Violent behavior, 2006, vol. 11, n. ${ }^{\circ}$ 3, pp. 237-264.

Carranza, Elías. "Situación del delito y de la Seguridad de los habitantes en los países de América Latina”, en ElíAs CARranza (coord.) Delito y seguridad de los habitantes. Costa Rica, ILANUD, Siglo XXI, 1997, pp. 23-49.

Ciudad Nuestra. Segunda Encuesta Metropolitana de Victimización 2012 - Resultados en 35 distritos de Lima, Lima, Ciudad Nuestra, 2012.

Comisión Nacional para el Desarrollo y Vida Sin Drogas (DEVIDA). Estrategia Nacional de Lucha contra las Drogas 2012-2016. Lima: Comisión Nacional para el Desarrollo y Vida Sin Drogas, 2016.

Contreras-Pulache, Hans et al. "Características de la violencia durante el embarazo en adolescentes de Lima, Perú", en Revista Peruana de Medicina Experimental y Salud Pública, vol. 30, n. . 3, 2013, pp. 379-385. 
De la Cuba, Pablo; Novak, Fabián y Namihas, SAndra. La trata de personas con fines de explotación laboral en el Perú. El caso de la minería aurífera y la tala ilegal de madera en Madre de Dios. Lima, Instituto de Estudios Internacionales (IDEI) de la Pontificia Universidad Católica del Perú y la Organización Internacional para las Migraciones (OIM), 2009.

De la Rosa. Paola y Sandoval Navarro, Víctor. "Los Sesgos Cognitivos y su Influjo en la decisión judicial. Aportes de la psicología jurídica a los procesos penales de corte acusatorio", en Revista Derecho Penal y Criminología, Volumen XXXVII, n. ${ }^{\circ}$ 102, pp. 141-164, Universidad Externado de Colombia, junio 2016.

Del Frate, Anna. "Crime and criminal justice statistics challenges", en STEFAN Harrendorf, MarkKu Heiskanen y Steven Malby (eds.). Internation Statistics of Justice and Crime. Helsinki, HEUNI, 2010 p. 168.

Diefenbach, Donald L. y D. West, Mark. "Violent crime and Poisson regression: A measure and a method for cultivation analysis", en Journal of Broadcasting \& Electronic Media, 2001, vol. 45, n. ${ }^{\circ}$, pp. 432-445.

Diefenbach, Donald L. y West, Mark D. "Violent crime and Poisson regression: A measure and a method for cultivation analysis", en Journal of Broadcasting \& Electronic Media, 2001, vol. 45, n. ${ }^{\circ}$ 3, pp. 432-445.

Díez Ripollés, José Luis. Estudios Penales y de Política Criminal, Lima: IDEMSA, 2007, pp. 55,

Dreze, JEAN y KHERA, ReETIKA. “Crime, gender, and society in India: Insights from homicide data", en Population and development review, 2000, vol. 26, n. ${ }^{\circ}$, pp. 335-352.

EMERICK, NiCHOLAR et al. "Homicide and social disorganization on the border: Implications for Latino and immigrant populations", Social Science Quarterly, Vol. 95, n. ${ }^{\circ}$ 2, Southwest Social Science Association, 2014, pp. 360-379.

Flake, Dallan F.. "Individual, family, and community risk markers for domestic violence in Peru", en Violence against women, vol. 11, n. . 3, 2005, pp. 353-373.

FLemming BALvig et al. "The public sense of justice in Scandinavia: A study of attitudes towards punishments", en European Journal of Criminology, vol. 12, no 3,pp. 342-361.

GARCÍA ESPAÑA, ELISA. Inmigración y delincuencia en España: análisis criminológico. Valencia, Tirant lo Blanch, Instituto Andaluz de Investigación Criminológica, 2001. 
GARTNer, Rosemary; BAKer, KATHRyn y PAMPEL, Fred. "Gender stratification and the gender gap in homicide", en Social Problems, 1990, vol. 37, n. ${ }^{\circ}$, pp. 593-612.

GonZÁlez VelázQuez. Rocío. "Cuando el derecho penal no basta. Reflexiones en torno a la tipificación del feminicidio en México", en Alegatos-Revista Jurídica de la Universidad Autónoma Metropolitana, May-Ago 2014, n. ${ }^{\circ}$ 87, pp. 271-308.

GURR, TED R.. "Historical trends in violent crime: A critical review of the evidence", en Crime and Justice, 1981, pp. 295-353.

Hernández Breña, Wilson. "Feminicidio (agregado) en el Perú y su relación con variables macrosociales", en URVIO-Revista Latinoamericana de Seguridad Ciudadana, n. ${ }^{\circ}$ 17, 2015, FLACSO - Ecuador (Facultad Latinoamericana de Ciencias Sociales), pp. 48-66.

Hernando Sanz, FeliPe. "Eclecticismo y Diversidad en la Geografía del Crimen y la Delincuencia en el Cambio de Siglo/Eclecticism and diversity in the geography of crime and delinquency in the turn of the century/Éclectisme et diversité dans la géographie du crime et la délinquance dans le changement de siècle", en Anales de geografía de la Universidad Complutense, Vol. 26, enero 2006, Universidad Complutense de Madrid, 2006, pp.9-30.

Instituto Nacional de Estadística e Informática- INEI, Perú. Estimaciones y proyecciones por sexo, según departamento, provincia y distrito 2000-2015, Lima, 2009.

Instituto Nacional de Estadística e Informática. Encuesta Nacional de Programas Estratégicos, Lima, INEI, 2016.

Lab, Steven et al. Criminal Justice: The Essentials, Oxford, Oxford University Press, 2013.

Land, Kenneth C.; McCall, Patricia L. y Cohen, Lawrence E. "Structural covariates of homicide rates: Are there any invariances across time and social space?", en American Journal of Sociology, Vol. 95, n. ${ }^{\circ}$ 4, Enero 1990, The University of Chicago Press, 1990, pp. 922-963.

LANDABURO SÁNCHEZ, LIOSDAY. Crimen organizado y economía ilegal. URVIO-Revista Latinoamericana de Estudios de Seguridad, 2016, n. ${ }^{\circ} 18$, p. 125-136.

LeVitT, Steven. "The Relationship between Crime Reporting and Police: Implications for the use of Uniform Crime Reports", en Journal of Quantitative Criminology, 1998, vol. 14, n. ${ }^{\circ}$, pp. 61-81. 
Loftin, Colin y Mcdowal, DAvid. "The Use of Official Records to Measure Crime and Delinquency", en Journal of Quantitative Criminology, 2010, vol. 26, n. ${ }^{\circ}$ 4, pp. 527-532

LÓPEZ, MARÍA et al. "Muertes por homicidio, consecuencia fatal de la violencia: El caso de México, 1979-1992”. Revista de Saúde Pública, 1996, vol. 30, n.1, p. 46-52.

Marchiori, Hilda. Criminología: La víctima del delito, México, Porrúa, 1998.

MAY CRIPE, SwEE et al. "Association of intimate partner physical and sexual violence with unintended pregnancy among pregnant women in Peru", en International Journal of Gynecology \& Obstetrics, vol. 100, n. ' 2, 2008, pp. 104-108.

Meloy, Michelle y Miller, Susan. The victimization of women: Law, policies, and politics. Nueva York, Oxford University Press, 2010.

Miller, Mitchell J. (ed.). The Encyclopedia of Theoretical Criminology, Vol. 1, Nueva Jersey, John Wiley \& Sons, 2014.

Ministerio de Justicia y Derechos Humanos del Perú. Homicidios en el Perú: contándolos uno a uno 2012. Lima: Ministerio de Justicia y Derechos Humanos, 2014.

Ministerio de Justicia y Derechos Humanos del Perú. Instituto Nacional de Estadística e Informática - Comité Estadístico Interinstitucional de la Criminalidad, Homicidios en el Perú: contándolos uno a uno 2011-2015. Lima, Instituto Nacional de Estadística e Informática, 2015.

Montero, Víctor. "Análisis psicosocial del discurso de la prensa sensacionalista peruana y las actitudes de sus lectores". Revista de investigación en psicología, 2008, vol. 11, n. ${ }^{\circ}$, pp. 153-181.

Mujica J., Jaris y Tuesta, Diego. "Problemas de construcción de indicadores criminológicos y situación comparada del feminicidio en el Perú", en Anthropologica, Vol. 30 n. . 30, Pontifica Universidad Católica del Perú, 2012, pp. 169-194.

Muñoz Cabrejo, Fanni. "Discursos sobre el feminicidio en la prensa escrita: El Comercio (2012) y Trome (2013): entre la visibilización/invisibilización del fenómeno", en Intersticios de la política y la cultura. Intervenciones latinoamericanas, 2016, vol. 5, n. ${ }^{\circ}$, pp. 97-125.

Naciones Unidas Oficina contra la Droga y el Delito (UnODC). Drogas y Delitos en el Perú: Situación actual y Evolución, Informe 2007. Lima: Naciones Unidas contra la Droga y el Delito, 2007. 
Nuñovero Cisneros, Lucía. "Política criminal actuarial y tráfico de drogas en el Perú de los noventas”, en Revista Internacional Derecho Penal Contemporáneo, Vol. 31, abr-jun. 2010, pp. 43-80. Bogotá, Legis, 2010.

O'Brien, RoBert. “Police productivity and crime rates: 1973-1992”, en Criminology, n. ${ }^{\circ} 34,1996$, p. 183. Colin Loftin y David Mcdowall, "The Use of Official Records to Measure Crime and Delinquency, Journal of Quantitative Criminology, 2010, vol. 26, no 4, pp. 527-532.

O'Carroll, Patrick W. y Mercy, James A. "Regional variation in homicide rates: why is the West so violent?", en Violence and victims, vol. 4, n. ${ }^{\circ}$ 1, 1989, pp. 17-25.

PATRICIA L. MCCALl et al. "Explaining variation in homicide rates across Eastern and Western European cities: the effects of social, political, and economic forces", en Handbook of European Homicide Research, Nueva York, Springer New York, 2012, pp. 137-154.

Odriozola, Enrique; Fernández Montalvo, Javier y Paz de Corral Gargallo. “Hay diferencias entre la violencia grave y la violencia menos grave contra la pareja?: un análisis comparativo", en International Journal of Clinical and Health Psychology, Vol. 8, n. ${ }^{\circ}$, Asociación Española de Psicología Conductual, 2008, pp. 355-382.

Oficina de las Naciones Unidas contra la Droga y el Delito (UNODC). Estudio Mundial sobre el homicidio 2013: Tendencias, contexto, datos.

Organización Mundial de la Salud (OMS). Estudio multipaís de la oms sobre salud de la mujer y violencia doméstica contra la mujer, primeros resultados sobre prevalencia, eventos relativos a la salud y respuestas de las mujeres a dicha violencia: resumen del informe, 2005.

OSBORne, RAQUEL. "De la 'violencia' (de género) a las 'cifras de la violencia': una cuestión política”, en EMPIRIA. Revista de Metodología de Ciencias Sociales, 2008, no 15 , p. 99-124.

Pedraza, Ricardo; Neira, Paola y Sabogal, Yahira G. "Muertes violentas intencionalmente producidas en Bogotá, 1997-2005: diferencias según el sexo", en Revista Colombiana de Psiquiatría, 2008, vol. 37, n. 3, p. 316-329.

PÉRez Guadalupe, José Luis. Faites y atorrantes. Una etnografía del penal de Lurigancho, Lima, Centro de Investigaciones Teológicas, 1994.

PÉREZ GuAdalupe, José Luis. La construcción social de la realidad carcelaria. Lima, Pontificia Universidad Católica del Perú, Fondo, 2000. 
RAMíREZ, BEATRIZ. "Cuando la muerte se explica por el género: problematizando la tipificación del feminicidio/femicidio”, en Gaceta Constitucional, vol. 45, Lima, Gaceta Jurídica, 2011, pp. 353-360.

Robe Shaw, Clifford; McKay, Henry Donald y S. Hayner, Norman. Juvenile delinquency and urban areas: A study of rates of delinquents in relation to differential characteristics of local communities in American cities. Chicago, University of Chicago Press, 1942.

Roxin, Klaus. Dogmática Penal y Política Criminal. Manuel Abanto Vásquez (trad.), Lima, IDEMSA, 1998, p. 25.

Salvatore, Ricardo; Aguirre, Carlos y Joseph, Gilbert (eds.). Crime and punishment in Latin America: law and society since late colonial times. Durham y Londres, Duke University Press, 2001.

SANSÓ-RUBERT, DANIEL. "Nuevas tendencias de organización criminal y movilidad geográfica. Aproximación geopolítica en clave de inteligencia criminal"/Tìtulo en Inglés: "New Tendencies in Criminal Organization and Geographical Mobility. Geopolitical Approach and Criminal Intelligence", en UNISCI Discussion Papers, 2016, no 41, p. 181.

Schacht, R., Ryan Rauch, Kristin y Mulder, Monique. “Too many men: the violence problem?”, en Trends in Ecology \& Evolution, vol. 29, n. ${ }^{\circ} 4,2014$, pp. 214-222.

Stamatel, JANET P.. "Explaining variations in female homicide victimization rates across Europe", en European Journal of Criminology, 2014, vol. 11, n. 5, pp. 578-600.

Thompson, Kenneth. Moral panics. Nueva York, Routledge, 1998.

VÁsquez GonzÁlez, Carlos y Urpina, Carles Soto. "El análisis geográfico del delito y los mapas de la delincuencia”, en Revista de Derecho Penal y Criminología, 2013, n. ${ }^{\circ}$ 9, Universidad Externado de Colombia, Bogotá, pp. 419-448.

Vélez GuZMÁn, YulianA. "Femicides in Medellin, 2010-2011: Conceptualization, characterization, and analysis", en Revista Criminalidad, 2012, vol. 54, n. ${ }^{\circ}$ 2, pp. 13-26.

Villanueva, Rocío y Ministerio Público. Homicidio y feminicidio en el Perú. Lima, Observatorio de Criminalidad del Ministerio Público, 2009. Observatorio de Igualdad de Género de América Latina y el Caribe - CEPAL. Informe anual 2013-2014. El enfrentamiento de la violencia contra las mujeres en América Latina y el Caribe, Santiago de Chile, Naciones Unidas, 2015. 